\title{
HEAT DEPOSITION IN POSITRON SOURCES FOR ILC*
}

\author{
V. Bharadwaj, R. Pitthan, J. Sheppard, H. Vincke, J. W. Wang, SLAC, Stanford, CA 94309, U.S.A.
}

\section{Abstract}

In the International Linear Collider (ILC) positron source, multi-GeV electrons or multi-MeV photons impinge on a metal target to produce the needed positrons in the resulting electromagnetic showers. The incoming beam power is hundreds of kilowatts. Various computer programs - such as FLUKA or MARS - can calculate how the incoming beam showers in the target and can track the particle showers through the positron source system. Most of the incoming energy ends up as heat in the various positron source elements. This paper presents results from such calculations and their impact on the design of a positron source for the ILC.

\section{INTRODUCTION}

The ILC is a proposed $\mathrm{e}^{+} \mathrm{e}^{-}$linear collider with nominal center-of-mass energies $0.5-1.0 \mathrm{TeV}$. The positron beams are provided by a positron source [1]. Table 1 shows the nominal parameters of such a source taken from the US Linear Collider Technical Options Study (USLCTOS) [2]. From this table two things are immediately obvious. First the incident beam powers for either an electron or photon drive beam are about the same, nominally $222 \mathrm{~kW}$ for photons and $280 \mathrm{~kW}(2$ targets $\mathrm{x} 140 \mathrm{~kW})$ for the electrons. Secondly only a small fraction of the incoming beam energy is deposited in the target itself. To see where the rest on the beam energy goes, simulations using FLUKA [3] were carried using a geometry representative of a positron source.

Table 1: Positron source target parameters.

\begin{tabular}{|l|c|c|}
\hline Parameter & $\boldsymbol{\gamma}$ beam & e- beam \\
\hline Pulse energy on target [kJ] & 44 & 28 \\
\hline Average power per target [kW] & 222 & 140 \\
\hline Spot size on target [mm] & 0.75 & 2.5 \\
\hline Target material & $\begin{array}{c}\text { Ti- } \\
\text { alloy }\end{array}$ & $\begin{array}{c}\text { W76 } \\
\text { Re24 }\end{array}$ \\
\hline Target thickness [r.l., (cm)] & 0.4 & 4.0 \\
\hline Target energy absorption [\%] & 8 & 14 \\
\hline Target Radius [m] & 0.80 & 1.0 \\
\hline Revolution Rate [rpm] & 1200 & 1200 \\
\hline Pulsed Temperature Rise $\left({ }^{\circ} \mathrm{C}\right)$ & 410 & 256 \\
\hline Number of targets/spares & $1 / 1$ & $2 / 3$ \\
\hline
\end{tabular}

\section{SIMULATION GEOMETRY}

Figure 1 shows a schematic of a generic positron source. The incoming drive beam $\left(\mathrm{e}^{-}\right.$or $\gamma$ ) hits a target, typically tungsten-rhenium alloy for electrons or titanium alloy for photons. In both cases the target is about $1.5 \mathrm{~cm}$

\footnotetext{
* Supported by United States Department of Energy through contracts DE-AC02-76SF00515.
}

thick. The produced positrons are focused and matched by a solenoidal field and captured and accelerated by an Lband RF system. At about $250 \mathrm{MeV}$, the positron beam is separated from the electrons and photons resulting from the electromagnetic showers and further accelerated and transported to the damping ring complex.

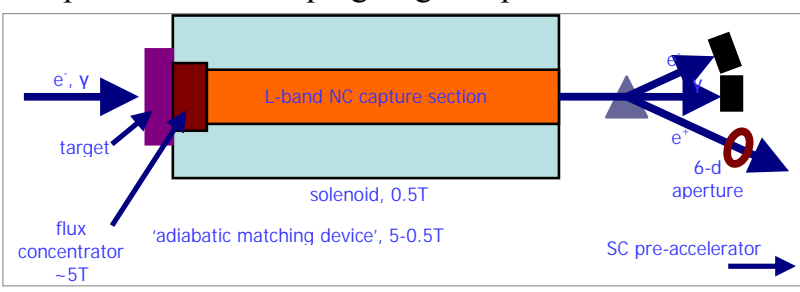

Figure 1: Generic positron source.

In order to simulate heat deposition for such a source, a cylindrical geometry was defined for input into FLUKA. Figure 2 shows a section of this geometry. Rotating this section $360^{\circ}$ around the horizontal (z) axis defines the "positron source". This source is finely segmented into rings in order to determine the distribution of energy depositions. The target is a thin segmented disk just before the $\mathrm{z}=0$ point on the horizontal axis and the beam hits the center of this disk.

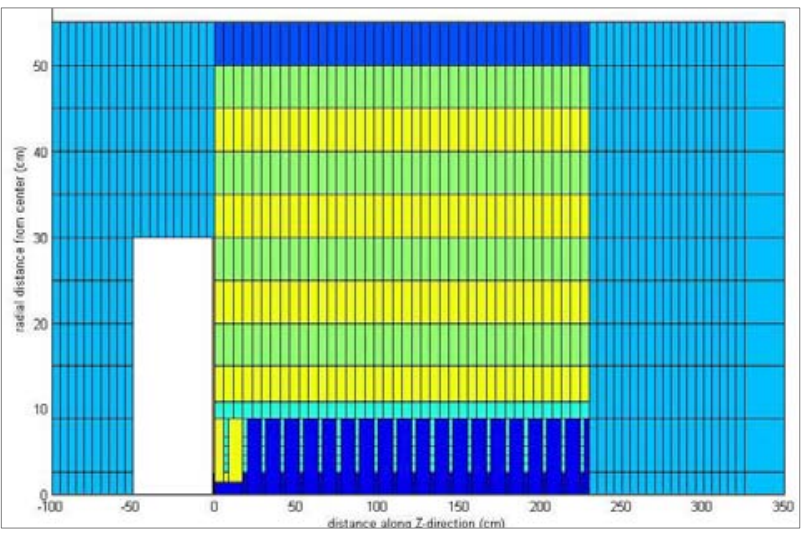

Figure 2: FLUKA simulation geometry.

There are three distinct regions, all cylinders $55 \mathrm{~cm}$ in radius, described by this geometry. The first is between $z$ $=-100-0 \mathrm{~cm}$. This region represents the pre-target shielding and contains the target itself. The target is a disk $30 \mathrm{~cm}$ in radius and either $1.35 \mathrm{~cm}$ (4.0 r.1.) of tungsten or $1.42 \mathrm{~cm}(0.4$ r.l.) of titanium. Before the target is a cylindrical air volume $30 \mathrm{~cm}$ in radius which is surrounded by iron "shielding". The innermost segments are disks $2.6 \mathrm{~cm}$ in radius. The rest of the shielding is segmented into rings all $5 \mathrm{~cm}$ thick in $\mathrm{z}$. Radially the rings dimensions are $r=2.6-8.9,8.9-15,15-20,20-25,25-30$, $30-35,35-40,40-45,45-50,50-55 \mathrm{~cm}$. The second region is the cylindrical iron volume between $\mathrm{z}=230-350$ $\mathrm{cm}$. This region represents the beam dump and shielding after the positron capture section. The segmentation is the 
same as the pre-beam shielding region, except for the last $\mathrm{z}$ segment which is $25 \mathrm{~cm}$ thick. The third region is just after the target and is contained in the cylindrical volume between $\mathrm{z}=0-230 \mathrm{~cm}$. This region represents the positron capture section and consists of RF cavities, the solenoid and solenoid shielding. In this region the segmentation in $\mathrm{z}$ is $5.75 \mathrm{~cm}$. Radially, the outermost set of rings, between $\mathrm{r}=50-55 \mathrm{~cm}$, consist of aluminum and represent the solenoid. Between $r=45-50,35-40,25-30,15-20 \mathrm{~cm}$ are sets of rings made of boron. Between $r=40-45,30$ $35,20-25,11.9-15 \mathrm{~cm}$ are sets of rings made of tungsten. These tungsten and boron rings represent the solenoid shielding.

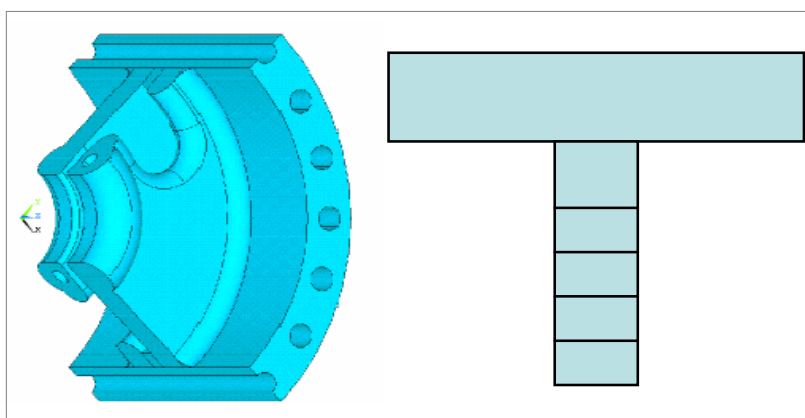

CAVITY DIMENSIONS

Inner iris radius $=26 \mathrm{~mm}$

Outer iris radius $=89 \mathrm{~mm}$

Iris spacing $\quad=115 \mathrm{~mm}$

Effective iris thickness (along $\mathrm{z}$ axis) $=26 \mathrm{~mm}$

Radial thickness of outer wall $=20 \mathrm{~mm}$

Number of cells in first two SW cavities $=20$

L-band copper structure with water cooling channels

FLUKA representation has the same average dimensions but is solid copper. The irises are segmented into five rings -

$\mathrm{r}=2.6-3.65,3.65-4.7,4.7-5.75,5.75--6.8,6.8-8.9 \mathrm{~cm}$

Figure 3: RF cavity and FLUKA representation.

\section{$R F$ Cavities}

In the third region the volume between $\mathrm{r}=0-11.9 \mathrm{~cm}$ represents the RF cavities. Reference [4] gives a description of what L-band RF systems for use with the ILC positron source are possible. Figure 3 show a section of an actual cavity design and its FLUKA representation. The walls of the cavity in FLUKA are segmented every $5.75 \mathrm{~cm}$ in $\mathrm{z}$ and the irises are segmented in $\mathrm{r}$ as indicated in Figure 3. The volume between the irises and the volume in the cylinder from $\mathrm{r}=0-2.6 \mathrm{~cm}$ is a vacuum. Additionally there can be a beam halo collimator after the target. This consists of tungsten which occupies the space from the target to the upstream face of the second RF iris - of course there is still a $2.6 \mathrm{~cm}$ radius hole in this collimator for the beam.

\section{FLUKA SIMULATION RUNS}

The beam parameters used for the conventional electron drive beam - simulation are as follows: beam energy $=6.2 \mathrm{GeV}(+/-0.1 \mathrm{GeV}$ gaussian $)$ beam size $=1 \mathrm{~mm}$ sigma, gaussian Average beam power $=280 \mathrm{~kW}$

Positron target $=1.35 \mathrm{~cm} / 4.0$ r.l. tungsten

For the undulator-based - photon drive beam simulation the beam parameters used were:

Beam energy $=6 \mathrm{MeV}(+/-4 \mathrm{MeV}$ flat dist)

Beam size $=1 \mathrm{~mm}$ sigma, gaussian

Average beam power $=220 \mathrm{~kW}$

Positron target $=1.42 \mathrm{~cm} / 0.4$ r.l. titanium

FLUKA runs were done for both photon and electron drives beam and with and without the collimators. Also a solenoidal field of 5 TESLA was applied to the entire region between $\mathrm{z}=0-230 \mathrm{~cm}$. It should be noted that FLUKA does not as yet have provision to model the effects of RF acceleration and there is no acceleration in any of the simulations. While this is a shortcoming in the simulation, it does not change the qualitative results of the calculations.

\section{ANALYSIS AND RESULTS}

\section{Heat Deposition in the RF Cavities}

Table 2 shows a summary of the results of the FLUKA simulations for heat deposition in the RF cavities.

Table 2: FLUKA simulations summary.

\begin{tabular}{|l|c|c|c|c|}
\hline & $\gamma$ beam & $\gamma+$ coll & e-beam & e- +coll \\
\hline \# incident particles & $2 * 10^{10}$ & $2 * 10^{10}$ & 5000 & 200 \\
\hline Beam E (MeV) & $2-10$ & $2-10$ & 6200 & 6200 \\
\hline Total Beam Power $(\mathrm{kW})$ & 220 & 220 & 280 & 280 \\
\hline Total L-band Power $(\mathrm{kW})$ & 13.48 & 3.00 & 148.42 & 97.72 \\
\hline Total Iris Power $(\mathrm{kW})$ & 9.79 & 2.33 & 125.13 & 84.91 \\
\hline Inner Irises Power $(\mathrm{kW})$ & 3.20 & 0.92 & 60.69 & 46.27 \\
\hline Collimator Power $(\mathrm{kW})$ & 0.00 & 12.02 & 0.0 & 54.37 \\
\hline Target Power Dep. $(\mathrm{KW})$ & 22.61 & 22.64 & 35.37 & 34.63 \\
\hline
\end{tabular}

The four columns of numbers are for the photon drive beam, the photon drive plus beam halo collimator, the electron drive beam, and the electron drive beam plus beam halo collimator respectively.

In the photon drive beam case, the shower products are very forward peaked and of the $220 \mathrm{~kW}$ of incoming beam power $22.6 \mathrm{~kW}-10.3 \%$ - of the beam power is deposited in the target and $13.5 \mathrm{~kW}-6.1 \%$ - is deposited in the copper RF structure. Of the beam power deposited in the RF structures $73 \%$ goes into the irises and $24 \%$ is deposited in the innermost $1.05 \mathrm{~cm}$ rings of the irises. Adding in the tungsten collimator improves the situation significantly, reducing these energy depositions by a factor of approximately four.

For the electron drive beam case the situation is markedly different. Of the $280 \mathrm{~kW}$ of incoming beam power $35.4 \mathrm{~kW}-12.6 \%$ - is deposited in the target, a 
number not too different that in the photon drive case. However, in the electron drive beam case the shower products coming out of the target have a much larger angular spread and the other energy deposition numbers are very different. The energy deposited in the RF is $148.4 \mathrm{~kW}-53 \%$ - of the incoming beam power of which $125.1 \mathrm{~kW}$ goes into the irises. The innermost $1.05 \mathrm{~cm}$ rings of the irises receive $60.9 \mathrm{~kW}$ of the incoming beam power. The addition of a tungsten collimator to the simulation improves the situation by absorbing about one third of the power exiting the target, not as much as the factor of four in the case of the photon drive beam. Nonetheless the power deposited in the L-band RF system is still almost $100 \mathrm{~kW}$ of which $46 \mathrm{~kW}$ is deposited in the innermost rings of the cavity irises.

The innermost iris ring - corresponding to $r=2.6-3.65$ $\mathrm{cm}$ - heat deposition power density is shown in Table 3 . The columns represent the iris \# (the RF cavity consists of 20 cells), the zmin and zmax of the iris, the power density for the photon drive beam with and without the collimator and the power density for the electron drive beam with and without the collimator.

Table 3: Innermost iris ring power densities.

\begin{tabular}{|c|c|c|c|c|c|c|}
\hline \multicolumn{3}{|c|}{ RING r=2.6-3.65 cm } & \multirow{2}{*}{\multicolumn{4}{|c|}{\begin{tabular}{c|c|c|}
$\gamma$ beam & $\gamma+$ coll & e-beam $\mid$ e- + coll \\
DEPOSITED POWER
\end{tabular}}} \\
\hline \multirow[t]{2}{*}{ IRIS \# } & \multirow{2}{*}{\begin{tabular}{|c|}
$\boldsymbol{z m i n}$ \\
$\mathrm{cm}$
\end{tabular}} & \multirow{2}{*}{\begin{tabular}{|c|}
$\boldsymbol{z m a x}$ \\
$\mathrm{cm}$ \\
\end{tabular}} & & & & \\
\hline & & & \multicolumn{4}{|c|}{$\mathrm{J} / \mathrm{cm}^{* *} 3 / \mathrm{sec}$} \\
\hline 1 & 5.75 & 8.35 & 27.578 & 1.799 & 84.552 & 6.908 \\
\hline 2 & 17.25 & & & & & \\
\hline 3 & & & & & & \\
\hline 4 & & & & & & \\
\hline 5 & & & & & & \\
\hline 6 & & & & & & \\
\hline 7 & & & & & & \\
\hline 8 & & & & & & \\
\hline 9 & & & & & & \\
\hline 10 & & & & & & \\
\hline 11 & & & & & & \\
\hline & & & & & & \\
\hline 13 & & & & & & \\
\hline 14 & & & & & & \\
\hline 15 & 5.75 & & & & & \\
\hline 16 & & & & & & \\
\hline 17 & 9.75 & & & & & \\
\hline & & & & & & \\
\hline & & & & & & \\
\hline 20 & 224.25 & 226.85 & 4.551 & 4.556 & 25.053 & 21.44 \\
\hline
\end{tabular}

It is seen that in the case of the photon drive beam most of the power in the irises is deposited in the first few irises and the beam halo collimator is very good at cleaning this up. However in the electron drive beam case the power is deposited more uniformly and significant power - and much more relative to the photon drive beam - is deposited along the entire length of the cavity. In this case the collimator cleans up the power deposition in the first couple of irises leaving the rest of the power deposition unaffected. The energy deposited in the RF structures has to be removed. In case of the electron drive beam, this is a significant constraint on the RF system design and the maximum accelerating gradient that can generated in the L-band RF system.

\section{Heat Deposition in the Solenoid}

The capture section of a positron source requires a solenoidal field. In order to determine whether a superconducting solenoid can be used to generate this field, an estimate of the heat deposition in such an object resulting from the drive beam hitting the target has to be made. The outer most cylinder in the third region $-\mathrm{z}=0-230 \mathrm{~cm}$ and $\mathrm{r}=50-55 \mathrm{~cm}-$ is made of aluminum and represents a solenoid. As described in the FLUKA geometry section, the RF cavity is surrounded by alternating layers of tungsten and boron to act as shielding for the solenoid.

Table 4: Losses in solenoid and its shielding.

\begin{tabular}{|l|c|c|}
\hline Region loss & $\boldsymbol{\gamma}$-beam & $\mathbf{e}^{-}$beam \\
\hline & $($ loss $W)$ & $($ loss $W)$ \\
\hline Solenoid & 0.29 & 0.12 \\
\hline $\mathrm{W}$ - shielding & 6,103 & 31,915 \\
\hline B - shielding & 5.3 & 71.9 \\
\hline Pre-beam dump & 782 & 220 \\
\hline & & \\
\hline Drive beam power $(W)$ & 220,000 & 280,000 \\
\hline
\end{tabular}

The results of the calculation are shown in Table 4. It is seen that the heat deposition in the "solenoid" can be made insignificant even with the simple shielding arrangement in the FLUKA geometry. Heat losses in the solenoid are less than one watt for incoming beam powers of a few hundred $\mathrm{kW}$. Such heat losses are easily handled by magnet cryogenics and a superconducting solenoid can be considered for the positron capture system. Table 4 also indicates that a fraction of one percent of the beam power comes back out of the target and this region can also be used for a (shielded) superconducting solenoid.

\section{REFERENCES}

[1] V. Bharadwaj et. al, "Design Issues for the ILC Positron Source" proceeding, this conference.

[2] "Accelerator Technology Options Report", 2004, URL http://www.slac.stanford.edu/xorg/accelops/

[3] A. Fassò, A. Ferrari, P.R. Sala, "Electron-photon transport in FLUKA: Status", MonteCarlo 2000, Lisbon, October 23--26 2000, p. 159-164 (2001).

A. Fassò, A. Ferrari, J. Ranft, P.R. Sala, "FLUKA: Status and Prospective for Hadronic Applications", MonteCarlo 2000, Lisbon, October 23--26 2000, p. 955-960 (2001).

[4] J. W. Wang et al., "Studies of Room Temperature Accelerator Structures for the ILC Positron Source", proceedings this conference. 